\title{
Enhancing the Sensitivity of Memory Tests: Reference Data for the Free and Cued Selective Reminding Test and the Logical Memory Task from Cognitively Healthy Subjects with Normal Alzheimer's Disease Cerebrospinal Fluid Biomarker Levels
}

Anna Brugulat-Serrat ${ }^{\mathrm{a}, \mathrm{b}, \mathrm{c}}$, Alba Cañas-Martínez ${ }^{\mathrm{a}}$, Lidia Canals-Gispert ${ }^{\mathrm{a}}$, Paula Marne $^{\mathrm{a}}$, Nina Gramunt ${ }^{\mathrm{d}}$, Marta Milà-Alomà ${ }^{\mathrm{a}, \mathrm{b}, \mathrm{c}, \mathrm{e}}$, Marc Suárez-Calvet ${ }^{\mathrm{a}, \mathrm{b}, \mathrm{c}, \mathrm{f}}$, Eider M. Arenaza-Urquijo ${ }^{\mathrm{a}, \mathrm{b}, \mathrm{c}}$, Oriol Grau-Rivera ${ }^{\mathrm{a}, \mathrm{b}, \mathrm{c}, \mathrm{f}}$, José María González-de-Echávarria ${ }^{\mathrm{a}}$, Carolina Minguillon ${ }^{\mathrm{a}, \mathrm{b}, \mathrm{c}}$, Karine Fauria ${ }^{\mathrm{a}, \mathrm{c}}$, Gwendlyn Kollmorgen ${ }^{\mathrm{g}}$, Ivonne Suridjan ${ }^{\mathrm{h}}$, Henrik Zetterberg ${ }^{\mathrm{i}, \mathrm{j}, \mathrm{k}, \mathrm{l}}$, Kaj Blennow ${ }^{\mathrm{i}, \mathrm{j}}$, Juan Domingo Gispert ${ }^{\mathrm{a}, \mathrm{b}, \mathrm{m}}$, José Luis Molinuevo ${ }^{\mathrm{a}, 1}$ and Gonzalo Sánchez-Benavides ${ }^{\mathrm{a}, \mathrm{b}, \mathrm{c}, *}$ for the ALFA study ${ }^{2}$ ${ }^{a}$ Barcelonaßeta Brain Research Center (BBRC), Pasqual Maragall Foundation, Barcelona, Spain

${ }^{\mathrm{b}}$ Hospital del Mar Medical Research Institute (IMIM), Barcelona, Spain

${ }^{\mathrm{c}}$ Centro de Investigación Biomédica en Red de Fragilidad y Envejecimiento Saludable (CIBERFES), Madrid, Spain

${ }^{\mathrm{d}}$ Pasqual Maragall Foundation, Barcelona, Spain

${ }^{\mathrm{e}}$ Universitat Pompeu Fabra, Barcelona, Spain

${ }^{\mathrm{f}}$ Servei de Neurologia, Hospital del Mar, Barcelona, Spain

g Roche Diagnostics GmbH, Penzberg, Germany

${ }^{\mathrm{h}}$ Roche Diagnostics International Ltd, Rotkreuz, Switzerland

${ }^{\mathrm{i}}$ Department of Psychiatry and Neurochemistry, Institute of Neuroscience and Physiology,

University of Gothenburg, Mölndal, Sweden

${ }^{\mathrm{j}}$ Clinical Neurochemistry Laboratory, Sahlgrenska University Hospital, Mölndal, Sweden

${ }^{\mathrm{k}}$ Department of Neurodegenerative Disease, UCL Institute of Neurology, Queen Square, London, United Kingdom ${ }^{1}$ UK Dementia Research Institute at UCL, London, United Kingdom

${ }^{\mathrm{m}}$ Centro de Investigación Biomédica en Red de Bioingeniería, Biomateriales y Nanomedicina, (CIBERBBN), Madrid, Spain

\footnotetext{
${ }^{1}$ Present address: H. Lundbeck A/S, Copenhagen, Denmark.

${ }^{2}$ The complete list of collaborators of the ALFA Study can be found in the acknowledgements section.

*Correspondence to: Gonzalo Sánchez-Benavides, Barcelona ßeta Brain Research Center (BBRC), Pasqual Maragall Foundation, Wellington 30, 08005 Barcelona, Spain. Tel.: +34933160990; Fax: +34 932275783; E-mail: gsanchezb@barcelonabeta.org.
} 


\begin{abstract}
.
Background: Cognitive performance of a given individual should be interpreted in the context of reference standards obtained in cognitively healthy populations. Recent evidence has shown that removing asymptomatic individuals with biomarker evidence of Alzheimer's disease pathology from normative samples increases the sensitivity of norms to detect memory impairments. These kind of norms may be useful for defining subtle cognitive decline, the transitional cognitive decline between normal cognition and mild cognitive impairment.

Objective: The present study aims to provide norms for the Free and Cued Selective Reminding Test (FCSRT) and the Logical Memory subtest of the Wechsler Memory Scale-IV in a sample of individuals aged 50-70 years with normal levels of amyloid- $\beta$ and tau cerebrospinal fluid (CSF) biomarkers.

Methods: The sample was composed of 248 individuals from the ALFA+ study with negative amyloid- $\beta$ and tau CSF biomarker levels. Regression-based norms were developed, including adjustments for age, education, and sex when applicable. Results: We found that education was associated with the performance in all the variables of both tests while age had a marginal effect only in the delayed free recall of the FCSRT. Sex was also related to the performance in the FCSRT, with women outperforming men. Equations to calculate z-scores and normative percentile tables were created. As compared with previously published norms the reference data presented were more sensitive but less specific, as expected.
\end{abstract}

Conclusion: The use of the norms provided in this work, in combination with the already published conventional norms, may contribute to detecting subtle memory impairment.

Keywords: Alzheimer's diseases, amyloid, biomarkers, cognition, memory, norms, sex

\section{INTRODUCTION}

Norms obtained from a cognitively unimpaired population are necessary to interpret any given score in a neuropsychological test. These reference data provide an objective framework that is critical in deciding if an individual's performance is within the normal range or is suggestive of impairment, that is, unexpectedly low for their sociodemographic characteristics. Age, education, and, in a few cases, sex adjustments, are routinely provided in normative data because of their well-known impact on cognitive performance. However, other variables, such as the presence of preclinical Alzheimer's disease (AD) in some of the individuals included in a reference group, may limit the sensitivity of the norms in detecting subtle impairments in elderly subjects. Of note, amyloid- $\beta(A \beta)$ positivity, which defines the presence of Alzheimer's pathologic changes [1], has an estimated prevalence of between $10 \%$ and $23 \%$ in individuals with normal cognition in the age range of 50-70 years [2]. Moreover, up to $44 \%$ of individuals at age $\geq 65$ years may also present evidence of either abnormal $A \beta$ levels, tau pathology or neurodegeneration [3].

AD pathology may affect cognitive performance even in cognitively healthy individuals. Mounting evidence suggests that $A \beta$ has a low but consistent impact on cognition in asymptomatic individuals that is mainly captured by memory tasks in both crosssectional $[4,5]$ and longitudinal studies [6-8]. In contrast with this amyloid effect, the influence of tau on cognition in unimpaired individuals is less clear. While in symptomatic AD stages tau pathology correlates far better than $A \beta$ load with cognitive outcomes [9], it seems to be mainly uncorrelated in cognitively healthy individuals. However, some sensitive memory paradigms have recently found relevant associations to tau levels in a sample of negative AD biomarkers cognitively normal individuals [10].

Recent studies observed that the norms derived from samples of individuals without present or future relevant cerebral pathologies increase the ability to detect preclinical $\mathrm{AD}$ and the predictive accuracy of future cognitive decline. One of the possible approaches to provide more sensitive normative data consists of robust norming, which entails excluding individuals that developed clinical dementia at follow-up. For example, Grober et al. [11] found that robust norming improves the detection of incident dementia compared to conventional norms, using both the Free and Cued Selective Reminding Test (FCSRT) and Wechsler Memory Scale IV Logical Memory (LM). Another useful approach consists of excluding individuals with altered AD biomarkers. In the BIOFINDER cohort, Borland et al. [12] recently observed that the cut-offs obtained after excluding individuals with altered cerebrospinal fluid (CSF) $\mathrm{A} \beta$, p-tau, cerebrovascular pathology, and neurofilament light measures were $6.2 \%$ to $19.9 \%$ stricter that those obtained from the total population. The application of such cut-offs to the entire cohort increased the sensitivity and the Youden index to identify cognitively unimpaired individuals in the preclinical 


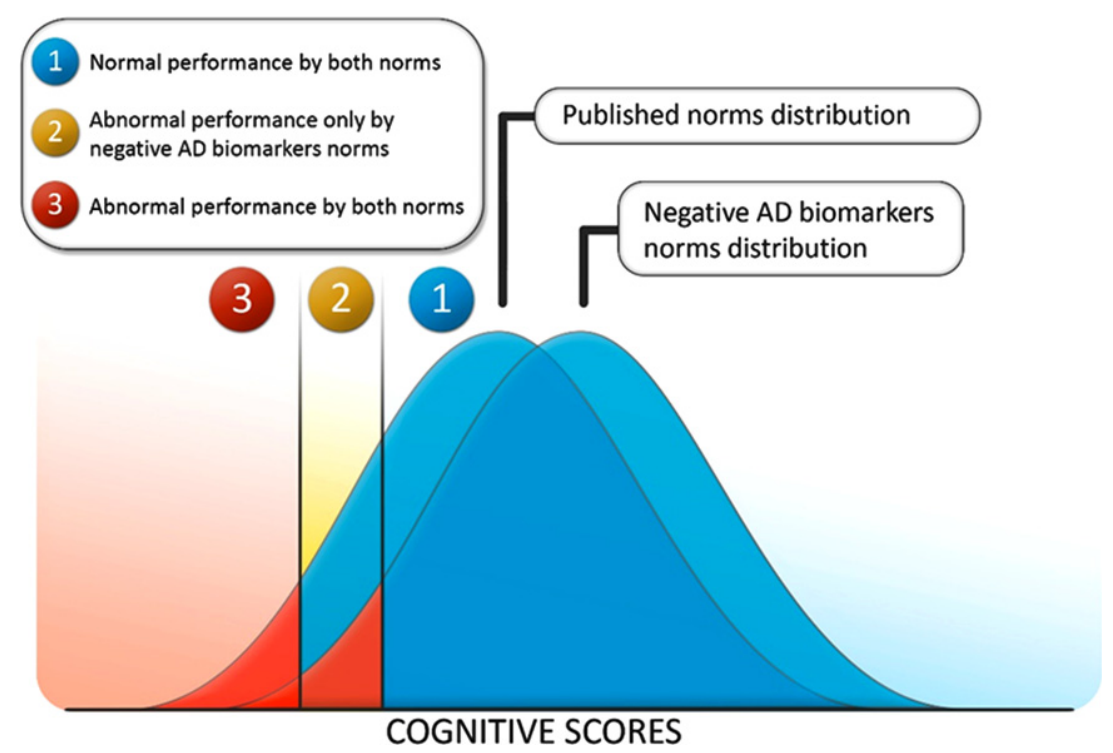

Fig. 1. Classification of cognitive performance obtained by combining conventional published norms and negative AD biomarkers norms (adapted from Bos et al. (2018) [13]).

AD stage. This effect was observed in all cognitive domains, except in naming, being the effect of AD pathology more pronounced for delayed memory scores and the effect of cerebrovascular disease more pronounced for executive measures. Another relevant study performed with pooled data from eight cohorts found that, compared with conventional published norms, the use of norms derived from $A \beta$-negative samples increased the predictive accuracy of future progression to dementia [13]. Such effect was found for memory measures (immediate and delayed recall of the Auditory Verbal Learning Test, AVLT), but not for verbal fluency or executive function (Trail Making Test) ones. As a result, a new group of individuals at higher risk of dementia was identified using $A \beta$ negative norms. Accordingly, the authors proposed a three-level model for interpreting memory scores by combining a "normal versus impaired" classification in both conventional published norms and $\mathrm{A} \beta$ negative norms (i.e., Group 1, normal with both norms; Group 2, impaired only with $A \beta$-negative norms; Group 3 impaired with both norms, Fig. 1).

Thus, having available normative data of memory measures obtained in individuals with evidence of absence of AD pathological changes can be useful to capture subtle cognitive difficulties that are missed by using conventional normative data. The main aim of the present study is to provide negative AD biomarker normative data for the FCSRT and LM subtest, which are widely used tests for the assessment of memory performance in individuals with suspected cognitive decline and have shown predictive validity for identifying individuals with memory complaints that will develop AD dementia [14].

\section{METHODS}

\section{Participants}

We included data from 248 participants that completed the first visit (2016-2019) from the ongoing ALFA+ (for ALzheimer and FAmilies) study. ALFA+ is a research cohort of middle-aged cognitively unimpaired subjects, many of whom are offspring of AD patients (in the present sample 153 out of 248 [61.7\%], had at least one parent diagnosed with AD before age 75), who have been deeply characterized by clinical interviews, lifestyle and risk factors questionnaires, cognitive testing, CSF biomarkers, and neuroimaging procedures, including magnetic resonance imaging (MRI), and A $\beta$ and FDG positron emission tomography (PET). All of these procedures are repeated every 3 years with the main aim of identifying the earliest pathophysiological changes in the preclinical AD continuum [15]. ALFA+ inclusion criteria were: 1) subjects who had previously participated in the 45-65/FPM2012 study (ALFA parent cohort [15]; 2) age between 45 and 75 years at the moment of the inclusion in the cohort (4565/FPM2012 study); 3) long-term commitment to 
the study: inclusion and follow-up visits and agreement to undergo all tests and study procedures (MRI, PET, and lumbar puncture). ALFA+ exclusion criteria included: 1) cognitive impairment (Clinical Dementia Rating [CDR] >0, Mini-Mental State Examination $[\mathrm{MMSE}]<27$, semantic fluency $<12$ ); 2 ) any significant systemic illness or unstable medical condition which could lead to difficulty complying with the protocol; 3) any contraindication to any test or procedure; 4) family history of monogenic AD. None of the individuals recruited was excluded due to cognitive impairment, being all the participants classified as cognitively unimpaired $(\mathrm{CDR}=0, \mathrm{MMSE}$ $\geq 27$ and semantic fluency $\geq 12$ ).

\section{AD biomarker status definition}

We used CSF analyses to define A $\beta$, p-tau, and total-tau status. CSF collection, processing, and storage in the ALFA+ study have been described previously [16]. CSF p-tau and t-tau were measured using the electrochemiluminescence Elecsys ${ }^{\circledR}$ PhosphoTau (181P) CSF and Total-Tau CSF immunoassays, respectively, on a fully automated cobas e 601 module (Roche Diagnostics International Ltd.). CSF $A \beta_{42}$ and $A \beta_{40}$ were measured with the exploratory Roche NeuroToolKit immunoassays (Roche Diagnostics International Ltd, Rotkreuz, Switzerland) on a cobas e 601 module. Measurements were performed at the Clinical Neurochemistry Laboratory, Sahlgrenska University Hospital, Mölndal, Sweden. A $\beta$ status $(A \beta+, A \beta-)$ was defined using the cutoff of 0.071 for the ratio $A \beta_{42 / 40}$. The p-tau cutoff used was $24 \mathrm{pg} / \mathrm{ml}$. The total-tau cutoff used was $300 \mathrm{pg} / \mathrm{ml}$ [16].

\section{Cognitive measures}

\section{Free and cued selective reminding test (FCSRT)}

The Spanish validated version A of the FCSRT was used in this study [17]. The FCSRT consists of the learning and retention of a list of 16 semantically unrelated words through a controlled learning process that uses semantic encoding. First, during learning, the participant is asked to read aloud 16 printed words ( 4 words in 4 cards) and associate them with their corresponding semantic cue (e.g., "Which is the bird?"). After this initial learning and encoding procedure, three recall trials are performed, each one preceded by $20 \mathrm{~s}$ of a number subtraction task. Each trial consists of free recall followed by cued recall for the words not spontaneously retrieved, by using the semantic cues previously given. The words that are not recalled after cueing are selectively reminded in the two initial trials, but not in the last one. A delayed free and cued recall is performed after $25-35 \mathrm{~min}$. For a complete description of the items used in FCSRT version A, see [18]. The main variables of the test are: the sum of the words correctly retrieved in the three free recall learning trials [Total Free Recall (TFR; range 0-48)]; the sum of the words recalled, either free or cued, in the three immediate recall trials [Total Recall (TR; range 0-48)]; the delayed free recall [Total Delayed Free Recall (TDFR; range 0-16)]; and the total amount of words recalled, either free or cued, in the delayed recall trial [Total Delayed Recall (TDR; range $0-16)]$.

\section{Logical memory (LM)}

The LM subtest used is included in the Wechsler Memory Scale-IV Spanish version [19]. It has three parts: immediate recall (LM I), delayed recall (LM II), and recognition (LM Recognition). In LM I, the examiner reads aloud two stories, and the examinee must reproduce the story immediately after hearing it as accurately as possible. After a period of between 20 and $30 \mathrm{~min}$, the examiner asks the participant to recall the two stories (LM II). In both parts, the memory score is computed by summing up the number of the remembered items for each story. Finally, a recognition task is performed, in which participants are given yes or no questions regarding details of the stories. In this study we used stories $\mathrm{B}$ and $\mathrm{C}$ regardless of age. The main variables are: Immediate Recall (LM I; range 0-50), Delayed Recall (LM II; range 0-50), and Recognition (range 0-30).

\section{Development of normative data}

To develop the normative data, we followed the regression-based method used in previous studies [20, 21]. In brief: 1) We centered the age of the participants by subtracting the mean group age from each subject's chronological age. 2) We constructed a set of multiple regression models (one for each cognitive score of interest), with cognitive score as dependent variable and age-centered, schooling (with 4 category levels [elementary $=0$, secondary $=1$, graduate $=2$, postgraduate $=3]$ ), and sex $($ male $=0$; female $=1)$ as predictors. A backward stepwise method was used, with a criterion of $p<0.1$ for the beta coefficient to maintain a predictor in the model. 3) We used the 
constant and the coefficients obtained to calculate predicted scores following Equation 1.

$$
\begin{aligned}
& \text { Predicted Score }=\text { Constant }+b_{1} * \text { Age centered } \\
& +b_{2} * \text { Schooling }+b_{3} * \text { Sex }
\end{aligned}
$$

4) We calculated the residuals between each possible value of the cognitive score and each possible expected score (using the relevant predictors for each variable) by subtracting them. Then, the residuals were converted to a $\mathrm{z}$-score by dividing them by the standard deviation of the unstandardized residuals of the regression model. Clinicians may use the equations with the coefficients provided in the results to calculate the z-score associated with a specific score of a given patient. 5) To simplify the use of the normative data, we provide tables for the most common percentiles (percentiles 1, 2, 5, 10, 15, 20, 30, 40, 50, $60,70,80,90,95,98)$. In each table, the theoretical raw scores associated with each percentile value are shown. When age accounted for a relevant effect in a cognitive variable, age groups were collapsed considering the distribution of the percentiles along the age range to reduce the number of tables.

\section{RESULTS}

Table 1 shows the demographic, genetic (APOE $\varepsilon 4$ allele), cognitive screening, and biomarker data of the present study's sample. Descriptive data of the memory tests are provided in Table 2.

The results of the multiple regression analysis with the estimated coefficient (beta) value for each variable and related $p$-value can be found in Table 3. Table 4 shows the equations used to calculate the z-scores by computing the discrepancy between the observed raw score and the predicted score accounting for relevant sociodemographic factors.

Normative tables with the calculations developed and raw scores equivalence to percentiles are provided in Supplementary Tables 1-8.

\section{DISCUSSION}

In this study, we provided regression-based normative data for the FCSRT and the LM memory tests obtained from a negative AD biomarker sample of cognitively healthy individuals aged between 50 and 70 years.

We found a relevant effect of schooling on the performance of both tasks. Sex affected the performance in three of the FCSRT variables, and age only
Table 1

Demographic, genetic, cognitive, and biomarker information of the sample $(n=248)$

\begin{tabular}{lccc}
\hline & Mean (SD) & Range & Count $(\%)$ \\
\hline Age & $60.5(4.5)$ & $50-70$ & \\
Sex (females) & & & $153(61.7 \%)$ \\
Education, y & $13.6(3.5)$ & $8-20$ & \\
Schooling* & & & \\
$\quad$ Elementary & & & $25(10.1 \%)$ \\
$\quad$ Secondary & & & $709(44 \%)$ \\
$\quad$ Graduate & & & $40(16.1 \%)$ \\
$\quad$ Postgraduate & & & $105(42.3 \%)$ \\
APOE 84 carriers & & & \\
MMSE & $29.2(0.9)$ & $27-30$ & \\
Animal fluency & $23.1(5.2)$ & $13-38$ & \\
A $\beta_{40}(\mathrm{ng} / \mathrm{mL})$ & $16.8(4.7)$ & $4.1-31.1$ & \\
A $\beta_{42}(\mathrm{pg} / \mathrm{mL})$ & $1474(513)$ & $383-3595$ & \\
A $\beta_{42 / 40}$ & $0.0865(0.0086)$ & $0.0711-0.1157$ & \\
p-tau (pg/mL) & $13.87(4.20)$ & $7.90-23.57$ & \\
t-tau (pg/mL) & $174.8(48.0)$ & $79.9-299.2$ & \\
\hline
\end{tabular}

* Schooling was recorded as follows: Elementary equals to finished elementary school (range of formal effective education 8-11 years); Secondary equals to finished secondary studies (range of formal effective education 9-14 years); Graduate equals to a university or superior degree (range of formal effective education 14-18 years); Postgraduate equals to Master or $\mathrm{PhD}$ (range of formal effective education 15-20 years). The CSF biomarkers cutoffs used were of 0.071 for the ratio $A \beta_{42 / 40}, 24 \mathrm{pg} / \mathrm{ml}$ for $\mathrm{p}$-tau and $300 \mathrm{pg} / \mathrm{ml}$ for total-tau [16].

Table 2

Memory tests descriptive data

\begin{tabular}{lcc}
\hline & Mean (SD) & Range \\
\hline FCSRT-TFR & $28.21(5.06)$ & $15-40$ \\
FCSRT-TR & $44.29(3.35)$ & $30-48$ \\
FCSRT-TDFR & $11.43(2.12)$ & $3-16$ \\
FCSRT-TDR & $28.21(5.06)$ & $10-16$ \\
LM I & $26.49(5.88)$ & $6-41$ \\
LM II & $22.45(6.24)$ & $2-36$ \\
LM Recognition & $25.17(2.86)$ & $15-30$ \\
\hline
\end{tabular}

FCSRT, Free and Cued Selective Reminding Test; TFR, Total Free Recall; TR, Total Recall; TDFR, Total Delayed Free Recall; TDR, Total Delayed Recall; LM, Wechsler Memory Scale-IV Logical Memory subtest; LM I, Immediate Recall; LM II, Delayed Recall.

in the FCSRT delayed free recall. Education is a wellknown factor associated with cognitive performance and should be always considered when interpreting cognitive data. For the FCSRT, the influence of education has been extensively reported and available norms offer education adjustments [17, 22, 23]. Similarly, education effects in the LM subtest have been consistently found by previous researchers in different countries and languages [23-25], but for this test, despite this evidence, the originally published norms only provide tables stratified by age. This 
Table 3

Results of the multiple regression analysis

\begin{tabular}{lccc}
\hline & Constant & Beta & $p$ \\
\hline FCSRT-TFR & 25.377 & & \\
Schooling & & 1.313 & $<0.001$ \\
Sex & & 1.340 & 0.039 \\
FCSRT-TR & 42.876 & & \\
$\quad$ Schooling & & 0.610 & 0.011 \\
$\quad$ Sex & & 0.779 & 0.074 \\
FCSRT-TDFR & 10.342 & & \\
$\quad$ Age (centered) & & -0.053 & 0.076 \\
$\quad$ Schooling & & 0.465 & 0.002 \\
$\quad$ Sex & & 0.613 & 0.025 \\
FCSRT-TDR & 14.855 & & \\
$\quad$ Schooling & & 0.204 & 0.013 \\
LM I & 23.875 & & \\
$\quad$ Schooling & & 1.710 & $<0.001$ \\
LM II & 19.380 & & \\
$\quad$ Schooling & & 2.010 & $<0.001$ \\
LM Recognition & 24.020 & & \\
$\quad$ Schooling & & 0.751 & $<0.001$ \\
\hline
\end{tabular}

FCSRT, Free and Cued Selective Reminding Test; TFR, Total Free Recall; TR, Total Recall; TDFR, Total Delayed Free Recall; TDR, Total Delayed Recall; LM, Wechsler Memory Scale-IV Logical Memory subtest; LM I, Immediate Recall; LM II: Delayed Recall.

fact limits the validity of those norms in less educated and highly educated individuals. The marginal effect of age found in this study was unexpected and may be attributable to the narrow age range of the individuals included. It is also possible that the inclusion of individuals with biomarker evidence of $\mathrm{AD}$ pathology in other normative data exacerbates the age-effect previously observed, because $\mathrm{AD}$ pathology is more prevalent in advanced ages. Regarding the sex effect, our findings for the FCSRT concur with those reported for the AVLT in the A $\beta$ negative norms developed by Bos et al. [13] and deserve a specific comment. A recent meta-analysis including 617 studies and more than 1.2 million healthy participants confirmed that women outperform men in all kinds of episodic memory tasks assessed, except in those involving spatial processing [26]. This sex effect, which is frequently dismissed in normative data, seems to have an impact on the diagnosis of mild cognitive impairment (MCI). Sundermann et al. [27] recently detected $10 \%$ of false negatives (missed MCIs) among females, and 10\% of false positives (non-MCI) among males when they used sex-specific norms for the AVLT. Furthermore, Banks et al. [28] found that sex-specific cognitive composites increase the statistical power and reduce the sample sizes needed in clinical trials. Accordingly, our sex and biomarker adjusted norms may be especially sensitive to diagnosing MCI among women.

Compared with published standard norms, the current norms can be described as more sensitive but less specific, because, as expected, after the removal of positive $\mathrm{AD}$ biomarkers individuals, the observed reference scores in this study are higher than those previously described. To illustrate the use of current norms compared with the previously published ones some examples are provided. We will consider performances $\leq$ percentile 5 as impaired. Example 1: A 65 -year-old male with a secondary degree of education (14 years) obtains a score of 42 in the immediate total recall of the FCSRT. This score corresponds to a percentile range between 29 and 40 according to the published Spanish norms [17], and a percentile range between 30 and 40 in the norms presented here. Thus, 42 is a normal performance in both norms (Group 1, according to Bos et al. nomenclature [13]). However, if the same individual obtains a score of

Table 4

Z-score calculation formula

\begin{tabular}{ll}
\hline FCSRT-TFR & (Raw score $-\left[25.377+\right.$ Schooling* $^{*} \cdot 1.313+$ Sex $\left.\left.^{\dagger} \cdot 1.34\right]\right) / 4.897$ \\
FCSRT-TR & (Raw score $-\left[42.876+\right.$ Schooling* $\cdot 0.61+$ Sex $\left.\left.^{\dagger} \cdot 0.779\right]\right) / 3.287$ \\
FCSRT-TDFR & (Raw score $-\left[10.342+\left(\right.\right.$ Age $\left.^{\ddagger}-60.5\right) \cdot(-0.053)+$ Schooling $* 0.465+$ Sex $\left.\left.^{\dagger} \cdot 0.613\right]\right) / 2.041$ \\
FCSRT-TDR & (Raw score $-[14.855+$ Schooling* 0.204$]) / 1.128$ \\
LM I & (Raw score $-[23.875+$ Schooling $* 1.71]) / 5.679$ \\
LM II & (Raw score $-[19.380+$ Schooling* 2.01$]) / 5.984$ \\
LM Recognition & (Raw score $-[24.020+$ Schooling* $\cdot 0.751]) / 2.785$ \\
\hline
\end{tabular}

${ }^{*}$ Schooling should be entered as: Elementary $=0$; Secondary $=1 ;$ Graduate $=2$; Postgraduate $=3 ;{ }^{\dagger}$ Sex should be entered as: Male $=0 ;$ Female $=1 .{ }^{\ddagger}$ Age should be centered to 60.5 . Elementary education equals to finished elementary school (range of formal effective education 8-11 years); Secondary equals to finished secondary studies (range of formal effective education 9-14 years); Graduate equals to a university or superior degree (range of formal effective education 14-18 years); Postgraduate equals to Master or $\mathrm{PhD}$ (range of formal effective education 15-20 years). FCSRT, Free and Cued Selective Reminding Test; TFR, Total Free Recall; TR, Total Recall; TDFR, Total Delayed Free Recall; TDR, Total Delayed Recall; LM, Wechsler Memory Scale-IV Logical Memory subtest; LM I, Immediate Recall; LM II, Delayed Recall. 
36 , this score corresponds to a percentile 11-18 in the published standard norms, but a percentile 2 in the current norms. In this case, performance is only impaired using the norms derived from the negative biomarker sample (Group 2). If such an individual obtains a score of 33, it would be impaired in both norms (percentile 3-5 and below 1, respectively, Group 3). The discrepancies between impaired scores according to the standard norms and according to the norms presented here would be even greater in the case of women, because they outperform men in almost 1 point in this variable, and the previously published norms do not adjust by sex. Example 2: A 60-year-old individual with an education equivalent to elementary studies scores 15 in the LM delayed recall (LM II). According to the norms published in the Spanish manual this corresponds to a percentile 37 , and percentile 20 according to the norms presented here, being within normal ranges in both cases. However, if the individual has postgraduate studies, the same score of 15 falls below percentile 5 in the negative AD biomarker norms and performance should then be considered as impaired, in clear discrepancy with the standard norms (percentile 37 in any case) which do not provide adjustments by education.

The approach involving concurrence or discrepancy of interpretations according to both types of norms, that is, using three categories rather than the dichotomous approach (preserved/impaired), may be useful to define the presence of the so-called "subtle cognitive decline". The definition of subtle cognitive decline remains elusive. The concept was incorporated in the National Institute on Aging-Alzheimer's Association (NIA-AA) research criteria for preclinical AD in 2011 [29]. In that framework, individuals with evidence of abnormal amyloid levels and neurodegeneration that present subtle cognitive decline, defined as a cognitive function that is "not normal, not MCI", were labeled as preclinical AD Stage 3 [29]. In the NIA-AA 2018 criteria, the numerical staging was restricted to the clinical expression of symptoms in the presence of underlying AD pathology, and subtle cognitive decline was related to the so-called transitional cognitive decline observable in the pre-MCI Stage 2 [1]. In both definitions of subtle cognitive decline, it can be documented either with subjective reports of cognitive decline (SCD) or by evidence of longitudinal objective cognitive decline. The usefulness of SCD to predict cognitive decline has been demonstrated. However, SCD may also be related to other medical conditions and most individuals with
SCD will not progress to dementia [30]. Regarding objective cognitive measures, although intraindividual longitudinal measures may be the most robust way of defining objective decline, some cross-sectional definitions of subtle cognitive decline have demonstrated utility to predict clinical progression in the ADNI cohort. Edmonds et al. in 2015 [31], following the concepts used for their actuarial definition of MCI, defined subtle cognitive decline as having at least two scores below 1 SD deviation in different cognitive domains, as opposed to the 1.5 SD cutoff usually used for endorsing cognitive impairment, or as having a slight functional decline in the Functional Activities Questionnaire (FAQ). The same research group further refined the definition of subtle cognitive decline by adding "process" scores in memory performance (i.e., learning slope, intrusion errors and retroactive interference [32]), and demonstrated that such definition of subtle cognitive decline related to faster amyloid accumulation and selective vulnerability of entorhinal cortical thinning [33]. Our approach, instead of using a more relaxed cutoff of $1 \mathrm{SD}$, suggests a complementary definition of subtle cognitive decline based on impairment (by using the common $<1.5$ SD or percentile 5 cutoffs), but using a reference group without evidence of AD pathology. Thus, we suggest that those performances falling in the Bos et al. Group 2 [13], that is, normal according to the published norms but impaired using negative AD biomarker ones, can be labelled as subtle cognitive impairment/decline. Although such an approach would eventually need negative biomarker norms for every cognitive test, the current evidence points out that only memory tasks would be affected by $\mathrm{AD}$ pathology in cognitively unimpaired individuals [13]. Furthermore, such norms would probably be progressively available using the open-access data from the large cohorts involving AD biomarkers collection that are currently under study.

The present study is not free of limitations. The main limitation relates to the limited applicability of the norms provided here. First, the narrow age range of the individuals included, from 50 to 70 years prevents their use on older individuals. However, our proposed definition of subtle cognitive decline by using the current norms may be especially useful in the age range between 60 and 70 , the age at which consultations to memory clinics for suspected cognitive decline highly increases. Moreover, these norms can be very useful in studies involving participants with preclinical $\mathrm{AD}$, in which detecting cognitive difficulties may be challenging. We also acknowl- 
edge that our sample is mainly composed of highly educated individuals, and the current norms would not be applicable to people who have not finished at least elementary studies. Also related to sample characteristics, it should be noted that there is a higher percentage of $A P O E \varepsilon 4$ carriers in our sample than in the general population. Although it can be argued that the APOE $\varepsilon 4$ allele may be associated with lower cognitive performance, we think that such an effect is controlled by incorporating AD biomarker measures, since $A P O E \varepsilon 4$ is a risk factor for $\mathrm{AD}$ and the cognitive effect of this allele is suggested to be mediated mainly through the presence of AD pathology [34]. The same rationale may be applied to the report of subjective cognitive decline in the sample (27\% of the sample expressed memory difficulties when asked) or to the presence of family history. It is also important to note the impact of the cut-offs used to define negative and positive $\mathrm{AD}$ biomarkers in the composition of the sample and the norms derived from it. While we used a highly sensitive cut-off using CSF biomarker levels [16], the use of other less specific cut-offs or the use of other measurements such PET imaging to define the reference group may lead to different norm distribution. Another limitation relates to the ceiling effect of the FCSRT, commonly observed in cognitively unimpaired or mildly impaired populations primarily in cued trials. The Memory Binding Test (MBT) was devised taking advantage of the FCSRT features to overcome such ceiling effect by using two lists of 16 paired words. We have previously provided some normative data from the ALFA cohort [35], and demonstrated the advantages of the MBT over the FCSRT [36]. However, despite having data available, we have not included MBT norms in the current paper because at the time of biomarker collection, participants had already been exposed to the MBT four years before and we have observed some trends of practice effects. In any case, robust MBT norms (calculated in those individuals without evidence of AD biomarkers or clinical decline at follow-up) will be published in short, along with extended normative data of the test.

A call to caution should be made to those researchers and clinicians who aim to use the current norms. These norms do not intend to replace the previously published ones. Instead, they may be used as a complementary interpretation framework. The selection of the most appropriate reference norms to interpret cognitive scores is an important decision in clinical neuropsychology. The current norms can be used in the cases that fit within the applicability range, that is, Spanish individuals with at least ele- mentary studies but mainly medium to high schooling and falling within the age range from 50 to 70 .

To summarize, we provided here regression-based norms for the FCSRT and the LM subtest developed in a sample of cognitively healthy individuals with evidence of negative CSF AD biomarkers. The current norms, in combination with the already published ones may be useful for detecting subtle memory impairment, especially in women.

\section{ACKNOWLEDGMENTS}

This publication is part of the ALFA study (ALzheimer and FAmilies). The authors would like to express their most sincere gratitude to the ALFA project participants and relatives without whom this research would have not been possible. Collaborators of the ALFA study are: Müge Akinci, Annabella Beteta, Raffaele Cacciaglia, Carme Deulofeu, Maria Emilio, Irene Cumplido, Ruth Dominguez, Carles Falcon, Sherezade Fuentes, Laura Hernandez, Gema Huesa, Jordi Huguet, Tania Menchón, Grégory Operto, Cleofé Peña-Gómez, Albina Polo, Sandra Pradas, Aleix Sala-Vila, Gemma Salvadó, Mahnaz Shekari, Anna Soteras, Laura Stankeviciute and Marc Vilanova.

The authors thank Roche Diagnostics International Ltd for providing the kits to measure CSF biomarkers, and the laboratory technicians at the Clinical Neurochemistry Lab in Mölndal, Sweden, who performed the analyses. COBAS, COBAS E, and ELECSYS are trademarks of Roche. The Roche NeuroToolKit is a panel of robust exploratory prototype assays used for research purposes only and not approved for clinical use.

The research leading to these results has received funding from "la Caixa" Foundation (LCF/PR/GN17/ 10300004) and the Alzheimer's Association and an international anonymous charity foundation through the TriBEKa Imaging Platform project (TriBEKa-17519007). Additional support has been received from the Universities and Research Secretariat, Ministry of Business and Knowledge of the Catalan Government under the grant no. 2017-SGR-892. MS-C received funding from the European Union's Horizon 2020 Research and Innovation Program under the Marie Sklodowska-Curie action grant agreement No 752310, and currently receives funding from the Spanish Ministry of Science, Innovation and Universities (Juan de la Cierva Programme grant IJC2018-037478-I). EMA-U is supported by the 
Spanish Ministry of Science, Innovation and Universities - Spanish State Research Agency (RYC2018026053-I) and is recipient of the Alzheimer's Association Research Grant (AARC 2019-AARG 644641). OG-R is supported by the Spanish Ministry of Science, Innovation and Universities (FJCI-201733437). JDG holds a 'Ramón y Cajal' fellowship (RYC-2013-13054).

Authors' disclosures available online (https:// www.j-alz.com/manuscript-disclosures/21-0640r2).

\section{SUPPLEMENTARY MATERIAL}

The supplementary material is available in the electronic version of this article: https://dx.doi.org/ 10.3233/JAD-210640.

\section{REFERENCES}

[1] Jack CR, Bennett DA, Blennow K, Carrillo MC, Dunn B, Haeberlein SB, Holtzman DM, Jagust W, Jessen F, Karlawish J, Liu E, Molinuevo JL, Montine T, Phelps C, Rankin KP, Rowe CC, Scheltens P, Siemers E, Snyder HM, Sperling R, Elliott C, Masliah E, Ryan L, Silverberg N (2018) NIAAA Research Framework: Toward a biological definition of Alzheimer's disease. Alzheimers Dement 14, 535-562.

[2] Jansen WJ, Ossenkoppele R, Knol DL, Tijms BM, Scheltens P, Verhey FRJ, Visser PJ, Aalten P, Aarsland D, Alcolea D, et al. (2015) Prevalence of cerebral amyloid pathology in persons without dementia: A meta-analysis. JAMA 313, 1924-1938.

[3] Jack CR, Wiste HJ, Weigand SD, Therneau TM, Knopman DS, Lowe V, Vemuri P, Mielke MM, Roberts RO, Machulda MM, Senjem ML, Gunter JL, Rocca WA, Petersen RC (2017) Age-specific and sex-specific prevalence of cerebral $\beta$-amyloidosis, tauopathy, and neurodegeneration in cognitively unimpaired individuals aged $50-95$ years: A cross-sectional study. Lancet Neurol 16, 435-444.

[4] Insel PS, Donohue MC, Sperling R, Hansson O, MattssonCarlgren N (2020) The A4 study: $\beta$-amyloid and cognition in 4432 cognitively unimpaired adults. Ann Clin Transl Neurol 7, 776-785.

[5] Jansen WJ, Ossenkoppele R, Tijms BM, Fagan AM, Hansson O, Klunk WE, Van Der Flier WM, Villemagne VL, Frisoni GB, Fleisher AS, Lleó A, Mintun MA, Wallin A, Engelborghs S, Na DL, Chételat G, Molinuevo JL, Landau SM, Mattsson N, Kornhuber J, Sabri O, Rowe CC, Parnetti L, Popp J, Fladby T, Jagust WJ, Aalten P, Lee DY, Vandenberghe R, De Oliveira CR, Kapaki E, Froelich L, Ivanoiu A, Gabryelewicz T, Verbeek MM, Sanchez-Juan P, Hildebrandt H, Camus V, Zboch M, Brooks DJ, Drzezga A, Rinne JO, Newberg A, De Mendonça A, Sarazin M, Rabinovici GD, Madsen K, Kramberger MG, Nordberg A, Mok V, Mroczko B, Wolk DA, Meyer PT, Tsolaki M, Scheltens P, Verhey FRJ, Visser PJ, Aarsland D, Alcolea D, Alexander M, Almdahl IS, Arnold SE, Baldeiras I, Barthel H, Van Berckel BNM, Blennow K, Van Buchem MA, Cavedo E, Chen K, Chipi E, Cohen AD, Förster S, Fortea J, Frederiksen
KS, Freund-Levi Y, Gkatzima O, Gordon MF, Grimmer T, Hampel H, Hausner L, Hellwig S, Herukka SK, Johannsen P, Klimkowicz-Mrowiec A, Köhler S, Koglin N, Van Laere K, De Leon M, Lisetti V, Maier W, Marcusson J, Meulenbroek O, Møllergård HM, Morris JC, Nordlund A, Novak GP, Paraskevas GP, Perera G, Peters O, Ramakers IHGB, Rami L, Rodríguez-Rodríguez E, Roe CM, Rot U, Rüther E, Santana I, Schröder J, Seo SW, Sorininen H, Spiru L, Stomrud E, Struyfs H, Teunissen CE, Vos SJB, Van Waalwijk Van Doorn LJC, Waldemar G, Wallin ÅK, Wiltfang J, Zetterberg H (2018) Association of cerebral amyloid- $\beta$ Aggregation with cognitive functioning in persons without dementia. JAMA Psychiatry 75, 84-95.

[6] Ho JK, Nation DA, Alzheimer's Disease Neuroimaging Initiative (2018) Neuropsychological profiles and trajectories in preclinical Alzheimer's disease. J Int Neuropsychol Soc 24, 693-702.

[7] Timmers T, Ossenkoppele R, Verfaillie SCJ, van der Weijden CWJ, Slot RER, Wesselman LMP, Windhorst AD, Wolters EE, Yaqub M, Prins ND, Lammertsma AA, Scheltens P, van der Flier WM, van Berckel BNM (2019) Amyloid PET and cognitive decline in cognitively normal individuals: The SCIENCe project. Neurobiol Aging 79, 50-58.

[8] Baker JE, Lim YY, Pietrzak RH, Hassenstab J, Snyder PJ, Masters CL, Maruff P (2017) Cognitive impairment and decline in cognitively normal older adults with high amyloid- $\beta$ : A meta-analysis. Alzheimers Dement (Amst) 6, 108-121.

[9] Nelson PT, Alafuzoff I, Bigio EH, Bouras C, Braak H, Cairns NJ, Castellani RJ, Crain BJ, Davies P, Tredici K Del, Duyckaerts C, Frosch MP, Haroutunian V, Hof PR, Hulette CM, Hyman BT, Iwatsubo T, Jellinger KA, Jicha GA, Kövari E, Kukull WA, Leverenz JB, Love S, Mackenzie IR, Mann DM, Masliah E, McKee AC, Montine TJ, Morris JC, Schneider JA, Sonnen JA, Thal DR, Trojanowski JQ, Troncoso JC, Wisniewski T, Woltjer RL, Beach TG (2012) Correlation of Alzheimer disease neuropathologic changes with cognitive status: A review of the literature. $J$ Neuropathol Exp Neurol 71, 362-381.

[10] Tort-Merino A, Olives J, León M, Peñaloza C, Valech N, Santos-Santos MA, Càmara E, Grönholm-Nyman P, Martínez-Lage P, Fortea J, Molinuevo JL, Sánchez-Valle R, Laine M, Rodríguez-Fornells A, Rami L (2019) Tau protein is associated with longitudinal memory decline in cognitively healthy subjects with normal Alzheimer's disease cerebrospinal fluid biomarker levels. J Alzheimers Dis 70, 211-225.

[11] Grober E, Mowrey W, Katz M, Derby C, Lipton RB (2015) Conventional and robust norming in identifying preclinical dementia. J Clin Exp Neuropsychol 37, 1098-106.

[12] Borland E, Stomrud E, Van Westen D, Hansson O, Palmqvist S (2020) The age-related effect on cognitive performance in cognitively healthy elderly is mainly caused by underlying AD pathology or cerebrovascular lesions: Implications for cutoffs regarding cognitive impairment. Alzheimers Res Ther 12, 30.

[13] Bos I, Vos SJB, Jansen WJ, Vandenberghe R, Gabel S, Estanga A, Ecay-Torres M, Tomassen J, den Braber A, Lleó A, Sala I, Wallin A, Kettunen P, Molinuevo JL, Rami L, Chetelat G, de la Sayette V, Tsolaki M, Freund-Levi Y, Johannsen P, Novak GP, Ramakers I, Verhey FR, Visser PJ (2018) Amyloid- $\beta$, tau, and cognition in cognitively normal older individuals: Examining the necessity to adjust for biomarker status in normative data. Front Aging Neurosci 10, 193. 
[14] Derby CA, Burns LC, Wang C, Katz MJ, Zimmerman ME, L'italien G, Guo Z, Berman RM, Lipton RB (2013) Screening for predementia AD: Time-dependent operating characteristics of episodic memory tests. Neurology $\mathbf{8 0}$, 1307-1314.

[15] Molinuevo JL, Gramunt N, Gispert JD, Fauria K, Esteller M, Minguillon C, Sánchez-Benavides G, Huesa G, Morán S, Dal-Ré R, Camí J (2016) The ALFA project: A research platform to identify early pathophysiological features of Alzheimer's disease. Alzheimers Dement (N Y) 2, 82-92.

[16] Milà-Alomà $M$, Salvadó $G$, Gispert JD, Vilor-Tejedor N, Grau-Rivera O, Sala-Vila A, Sánchez-Benavides G, Arenaza-Urquijo EM, Crous-Bou M, González-deEchávarri JM, Minguillón C, Fauria K, Simon M, Kollmorgen G, Zetterburg H, Blennow K, Suárez-Calvet M, Molinuevo JL (2020) Amyloid- $\beta$, tau, synaptic, neurodegeneration and glial biomarkers in the preclinical stage of the Alzheimer's continuum. Alzheimers Dement 16, 13581371.

[17] Peña-Casanova J, Gramunt-Fombuena N, Quiñones-Úbeda S, Sánchez-Benavides G, Aguilar M, Badenes D, Molinuevo JL, Robles A, Barquero MS, Payno M, Antúnez C, Martínez-Parra C, Frank-García A, Fernández M, Alfonso V, Sol JM, Blesa R (2009) Spanish multicenter normative studies (NEURONORMA project): Norms for the ReyOsterrieth Complex Figure (copy and memory), and Free and Cued Selective Reminding test. Arch Clin Neuropsychol 24, 371-393.

[18] Grau-Guinea L, Pérez-Enríquez C, García-Escobar G, Arrondo-Elizarán C, Pereira-Cutiño B, Florido-Santiago M, Piqué-Candini J, Planas A, Paez M, Peña-Casanova J, Sánchez-Benavides G (2018) Development, equivalence study, and normative data of version B of the Spanish-language Free and Cued Selective Reminding Test. Neurologia 36, 353-360.

[19] Wechsler D (2013) Wechsler Memory Scale-IV. Spanish edition, Pearson Clinical \& Talent Assessment, Madrid.

[20] van der Elst W, van Boxtel MPJ, van Breukelen GJP, Jolles J (2006) Normative data for the Animal, Profession and Letter M Naming verbal fluency tests for Dutch speaking participants and the effects of age, education, and sex. J Int Neuropsychol Soc 12, 80-89.

[21] Guàrdia-Olmos J, Peró-Cebollero M, Rivera D, ArangoLasprilla JC (2015) Methodology for the development of normative data for ten Spanish-language neuropsychological tests in eleven Latin American countries. Neurorehabilitation 37, 493-9.

[22] Girtler N, De Carli F, Amore M, Arnaldi D, Bosia LE, Bruzzaniti C, Cappa SF, Cocito L, Colazzo G, Ghio L, Magi E, Mancardi GL, Nobili F, Pardini M, Picco A, Rissotto R, Serrati C, Brugnolo A (2015) A normative study of the Italian printed word version of the free and cued selective reminding test. Neurol Sci 36, 1127-1134.

[23] Vogel A, Stokholm J, Andreasen R, Henriksen BD, Brønniche V, Madsen GJ, Gustafsson M, Overgaard S, Guldberg AM, Jørgensen K (2018) Psychometric properties and reference data for Danish versions of Free and Cued Selective Reminding Test, Category Cued Memory Test and Logical Memory. Scand J Psychol 59, 496-502.

[24] Ahn YD, Yi D, Joung H, Seo EH, Lee YH, Byun MS, Lee JH, Jeon SY, Lee J-Y, Sohn BK, Lee DY (2019) Normative data for the Logical Memory Subtest of the Wechsler Memory Scale-IV in middle-aged and elderly Korean people. Psychiatry Investig 16, 793-799.
[25] O'Bryant SE, Edwards M, Johnson L, Hall J, Gamboa A, O'jile J (2018) Texas Mexican American adult normative studies: Normative data for commonly used clinical neuropsychological measures for English- and Spanishspeakers. Dev Neuropsychol 43, 1-26.

[26] Asperholm M, Högman N, Rafi J, Herlitz A (2019) What did you do yesterday? A meta-analysis of sex differences in episodic memory. Psychol Bull 145, 785-821.

[27] Sundermann EE, Maki P, Biegon A, Lipton RB, Mielke MM, Machulda M, Bondi MW (2019) Sex-specific norms for verbal memory tests may improve diagnostic accuracy of amnestic MCI. Neurology 93, e1881-e1889.

[28] Banks SJ, Shifflett B, Berg JL, Sundermann E, Peavy G, Bondi MW, Edland SD (2019) Sex-specific composite scales for longitudinal studies of incipient Alzheimer's disease. Alzheimers Dement (N Y) 5, 508-514.

[29] Sperling R a, Aisen PS, Beckett LA, Bennett D a, Craft S, Fagan AM, Iwatsubo T, Jack CR, Kaye J, Montine TJ, Park DC, Reiman EM, Rowe CC, Siemers E, Stern Y, Yaffe K, Carrillo MC, Thies B, Morrison-Bogorad M, Wagster M V, Phelps CH, Jack Jr CR, Kaye J, Montine TJ, Park DC, Reiman EM, Rowe CC, Siemers E, Stern Y, Yaffe K, Carrillo MC, Thies B, Morrison-Bogorad M, Wagster M V, Phelps CH, Jack CR (2011) Toward defining the preclinical stages of Alzheimer's disease: Recommendations from the National Institute on Aging-Alzheimer's Association workgroups on diagnostic guidelines for Alzheimer's disease. Alzheimers Dement 7, 280-292.

[30] Jessen F, Amariglio RE, Buckley RF, van der Flier WM, Han Y, Molinuevo JL, Rabin L, Rentz DM, Rodriguez-Gomez O, Saykin AJ, Sikkes SAM, Smart CM, Wolfsgruber S, Wagner $M(2020)$ The characterisation of subjective cognitive decline. Lancet Neurol 19, 271-278.

[31] Edmonds EC, Delano-Wood L, Galasko DR, Salmon DP, Bondi MW (2015) Subtle cognitive decline and biomarker staging in preclinical Alzheimer's disease. J Alzheimers Dis 47, 231-242.

[32] Thomas KR, Edmonds EC, Eppig J, Salmon DP, Bondi MW (2018) Using neuropsychological process scores to identify subtle cognitive decline and predict progression to mild cognitive impairment. J Alzheimers Dis 64, 195-204.

[33] Thomas KR, Bangen KJ, Weigand AJ, Edmonds EC, Wong CG, Cooper S, Delano-Wood L, Bondi MW, Alzheimer's Disease Neuroimaging Initiative (2020) Objective subtle cognitive difficulties predict future amyloid accumulation and neurodegeneration. Neurology 94, e397-e406.

[34] O'Donoghue MC, Murphy SE, Zamboni G, Nobre AC, Mackay CE (2018) APOE genotype and cognition in healthy individuals at-risk of Alzheimer's disease: A review. Cortex 104, 103-123.

[35] Gramunt N, Buschke H, Sánchez-Benavides G, Lipton RB, Peña-Casanova J, Diéguez-Vide F, Masramon X, Gispert JD, Fauria K, Camí J, Molinuevo JL (2015) Reference data of the Spanish Memory Binding Test in a midlife population from the ALFA STUDY (Alzheimer's and Family). $J$ Alzheimers Dis 48, 613-625.

[36] Gramunt N, Sánchez-Benavides G, Buschke H, Lipton RB, Masramon X, Gispert JD, Peña-Casanova J, Fauria K, Molinuevo JL (2016) Psychometric properties of the Memory Binding Test: Test-retest reliability and convergent validity. J Alzheimers Dis 50, 999-1010. 